focus $F^{\prime}$ is got very easily since $A^{\prime} F$ and $A^{\prime} F^{\prime \prime}$ are equally inclined to $A^{\prime} T$.

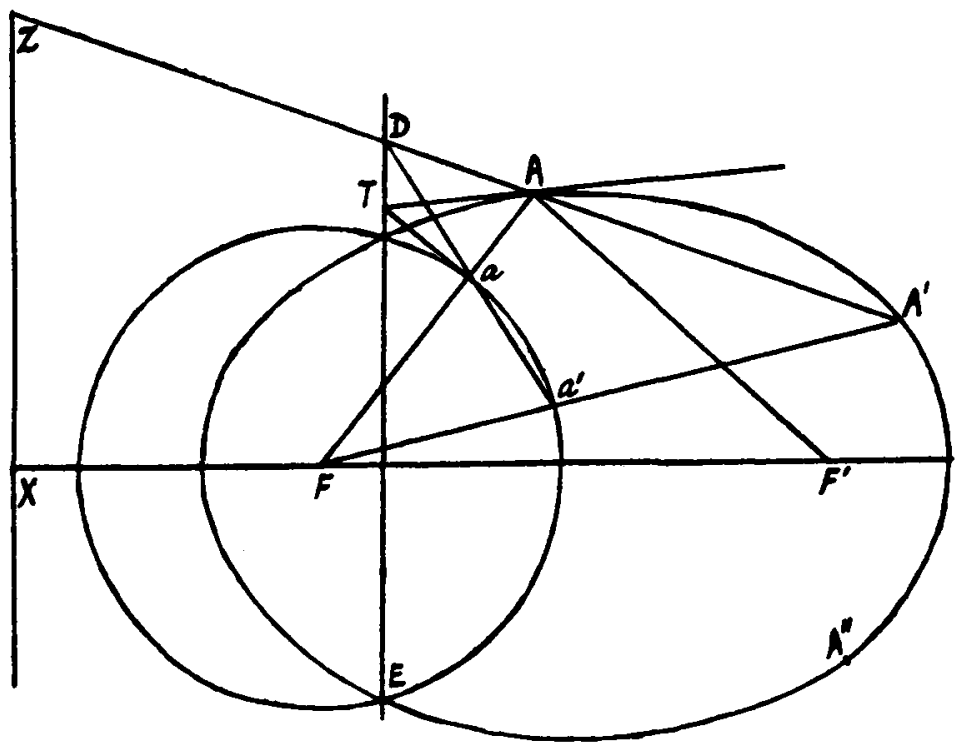

[See Housel's Introduction à la Géométrie Supérieure; Paris, Ganthiers-Villars, 1865, where a somewhat difficult proof depending on homology by de Jonquières is given.]

R. F. DAvis.

\title{
Common Logarithms calculated by simple multiplication.
}

The 4-place tables inform us that $\log 3=\cdot 4771$. This means that $3=10^{6071}$ or $3^{30000}=10^{4771}$; in other words, that there are $4771+1$ digits in $3^{10000}$. Hence to find $\log 3$ we have merely to raise 3 to the 10000 th power and count the digits in the result. This need not be so long a process as one might anticipate, if we use contracted multiplication, and arrange the work suitably. The figures given below form the actual calculation, which took 
about 10 minutes to complete, and could be done in a shorter time.

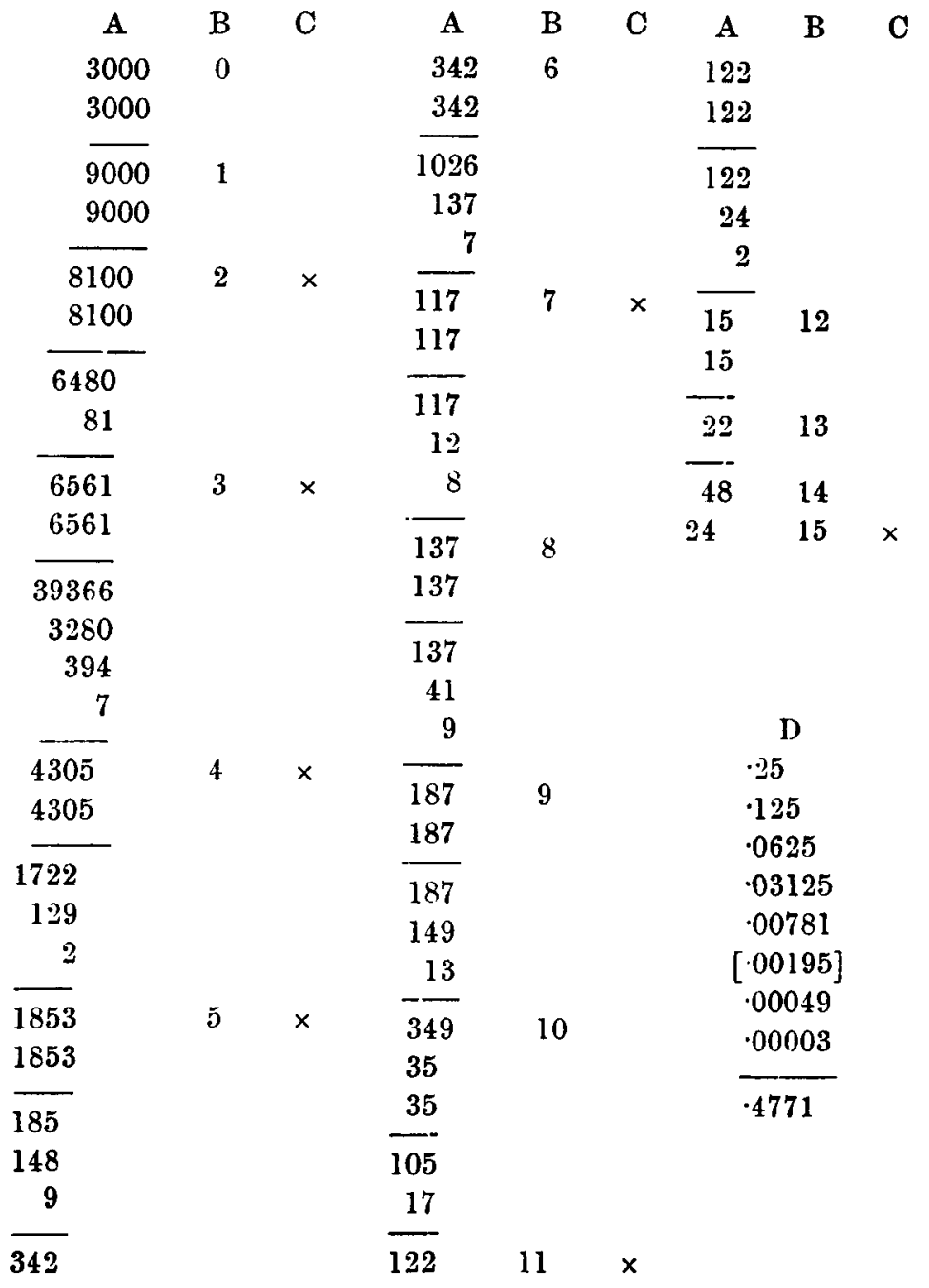

The process consists in squaring 3, squaring the result, and so on to the 15 th step which gives $3^{2}$, i.e. $3^{32768}$ which is more than sufficient. 
Of course we use contracted multiplication, carrying the calculation to 4 digits at first, afterwards to 3 and 2 digits. Column A gives the actual multiplication. Column $B$ gives the number of the step. Column $C$ has a mark $\times$ at each step where an extra digit appears on the left. The theory of this arrangement of the work may be thus explained .

$$
\begin{aligned}
& \log 3=\frac{1}{2} \log 9=\frac{1}{2^{2}} \log 81=\frac{1}{2^{2}}(1+\log 8 \cdot 1) \\
& =\frac{1}{2^{2}}+\frac{1}{2^{3}} \log 65 \cdot 61=\frac{1}{2^{2}}+\frac{1}{2^{3}}(1+\log 6 \cdot 561) \\
& =\frac{1}{2^{2}}+\frac{1}{2^{3}}+\frac{1}{2^{3}} \log 6 \cdot 561 \text { and so on, }
\end{aligned}
$$

so that $\log 3=\cdot 25+\cdot 125+\cdot 125 \log 6 \cdot 561$, etc., so that we have simply to add certain powers of 5 , viz., the $2 \mathrm{nd}, 3 \mathrm{rd}, 4 \mathrm{th}, 5 \mathrm{th}, 7 \mathrm{th}$, 11 th and 15 th to get the result. This is done in the sum marked $D$. Note that in calculating the 11 th power of 5 from the 7 th we have to divide by $2^{4}$, i.e. by $4 \times 4$ and it is convenient to do it in two steps, crossing out the intermediate quotient 00195 , which is to be left out in adding. If several logarithms had to be calculated it would be convenient to have the powers of $\frac{1}{2}$ expressed decimally in a column and simply cross out in pencil those not to be added.

Is it too much to ask that every class that uses logarithms should calculate a 4-place table for itself? Of course it would be unnecessary to calculate the 1000 logarithms separately. Half a dozen or fewer would suffice, then a sufficient number of others could be got as products, or fractions whose numerator and denominator are products of the numbers for which the logarithms have been calculated, to enable the rest to be filled in by "proportional parts."

To ensure the correctness of 4 digits, it would be advisable to begin by working to 5 places. Since $2^{10}=1024$ it is clear that we may reduce the number of places in our calculation by one for every three or four steps as has been done in the above example.

A more formal proof of the rule might be stated as follows:

Let $N$ be a number lying between 1 and 10 , so that the characteristic of $\log N$ is 0 and its mantissa $m$. Let $m_{1}$ be the mantissa of $2 \log N, m_{2}$ that of $2^{2} \log N, m_{r}$ that of $2^{r} \log N$. 
Then $m=\frac{1}{2}\left(c_{1}+m_{1}\right), m_{1}=\frac{1}{2}\left(c_{2}+m_{2}\right), m_{2}=\frac{1}{2}\left(c_{3}+m_{3}\right)$ and so on, where $c_{r}$ is either 1 or 0 according as the square of antilog $m_{r-1}$ is greater or less than 10.

Thus $m=\frac{c_{1}}{2}+\frac{c_{2}}{2^{2}}+\frac{c_{s}}{2^{3}}+\ldots+\frac{c_{n}}{2^{n}}+\frac{m_{n}}{2^{n}}$.

R. F. Muirhead.

\section{Trigonometrical Ratios of $(A \pm B)$.}

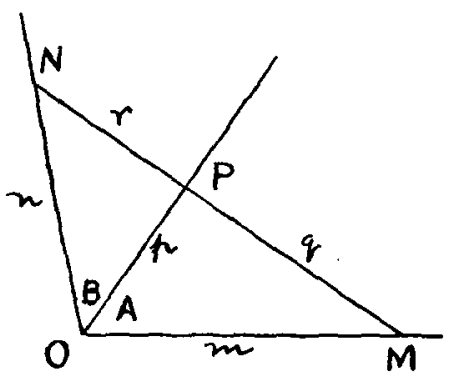

Fig. 1

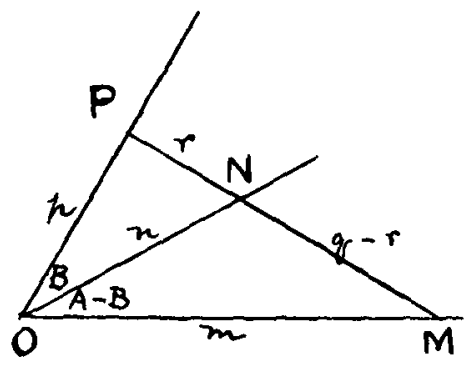

Fig. 2 .

The formulae for $\sin (A \pm B)$ and $\cos (A \pm B)$ may be derived by the following method, the main attraction of which is the simplicity of the figures employed.

Consider the case in which $A$ and $B$ are both acute angles.

Let $\angle M O P=A$ and $\angle P O N=B$, then in Fig. $1-M O N=A+B$ and in Fig. $2-M O N=A-B$. Through any point $P$ on $O P$, the arm common to both angles, draw a perpendicular to $O P$ meeting the other arms in $M$ and $N$ respectively.

Let $O M=m, O N=n, O P=p, M P=q, P N=r$.

From the triangle $O M N$ we obtain

$$
\begin{aligned}
\sin (A \pm B)=\sin M O N & =\frac{2 \triangle O M N}{m n}=\frac{p(q \pm r)}{m n} \\
& =\frac{q}{m} \cdot \frac{p}{n} \pm \frac{p}{m} \cdot \frac{r}{n} \\
& =\sin A \cos B \pm \cos A \sin B .
\end{aligned}
$$

